\section{Oropharynx-Ca: Durch PET-CT weniger Eingriffe}

\author{
Bei Patienten mit Kopf-Hals-Tumoren im Stadium N2 ist die Strategie einer \\ Überwachung per PET-CT einer geplanten Neck Dissection vor oder nach einer \\ Chemoradiotherapie nicht unterlegen. Mithilfe der Bildgebung werden aber \\ signifikant weniger Eingriffe vorgenommen.
}

\begin{abstract}
m den Nutzen der PET-CT-Stratifizierung zur Überwachung von Patienten mit Kopf-Hals-Tumoren im Vergleich zu einer Neck Dissection zu untersuchen, haben britische Onkologen um Prof. Hisham Mehanna von der Universität Birmingham in Großbritannien eine prospektive randomisierte Studie durchgeführt, an der zwischen 2007 und 2012 insgesamt 564 Patienten im Alter von durchschnittlich 58 Jahren teilnahmen. Primärer Endpunkt der Studie war das Gesamtüberleben.

Mit einem Anteil von über $80 \%$ hatten die meisten Patienten ein OropharynxKarzinom. Bei 17 \% lag ein Kopf-Hals-Tumor im Nodalstadium N2a vor, bei $61 \%$ im Stadium N2b, also kleiner als $6 \mathrm{~cm}$.
\end{abstract}

len Nachbeobachtungszeit von fünf Jahren.

Fazit: Wie die Ärzte berichten, musste nur bei 54 Patienten (19\%) in der Gruppe mit Bildgebung danach eine Neck Dissection vorgenommen werden. In der Vergleichsgruppe wurde der Eingriff dagegen bei 221 Patienten (78\%) gemacht. Die Überlebensrate war in beiden Gruppen etwa gleich groß $(84,9 \%$ in der Gruppe mit Bildgebung, $81,5 \%$ in der Vergleichsgruppe). Die Berechnung der Hazard Ratio (HR) für den Parameter Tod ergab einen leichten Vorteil für die Strategie mit Überwachung durch die Bildgebung (HR; 0,92; $95 \%$-Konfidenzintervall zwischen 0,65 und 1,$32 ; \mathrm{p}=0,004$ für Nichtunterlegenheit). Nach fünf Jahren waren 60 Patienten gestorben, in der Kontrollgruppe 62.

Peter Leiner

Dissection vorgenommen. In der Vergleichsgruppe erfolgte der Eingriff vier Wochen vor oder nach dem Ende der Radiochemotherapie. Das mediane Followup betrug 36 Monate, mit einer maxima-
Mehanna $\mathrm{H}$ et al. PET-CT Surveillance versus Neck Dissection in Advanced Head and Neck Cancer. NEJM 2016: online 23. März. doi: 10.1056/NEJMoa1514493

\title{
HNSCC: Lymphknoten mit Vorhersagekraft
}

\section{Die Analyse des Lymphknotenbefalls bei Patienten mit einem Platten- epithelkarzinom des Kopf- und Halsbereiches (HNSCC) gilt als wichtiger prognostischer Faktor, der die Therapieentscheidung mitbeeinflusst. Nun wurde untersucht, ob der Grad des Lymphknotenbefalls auch das Über- leben vorhersagt.}

B ei mehr als der Hälfte der Patienten mit einem HNSCC sind die Lymphknoten zum Diagnosezeitpunkt bereits von Tumorzellen befallen. Der Lymphknotenbefall verschlechtert die Überlebenschancen und gilt als einer der wichtigsten Faktoren für die Wahl der Therapie. Doch obwohl in vielen Studien bestätigt wurde, dass die Zahl der befallenen Lymphknoten ein wichtiger prognostischer Faktor für das lokal fortgeschrittene HNSCC ist, war bisher unbekannt, wie sich das Ausmaß des Lymphknotenbefalls auf das Überleben der Patienten auswirkt.

Nun wurden fast 15.000 Probanden aus dem amerikanischen SEER (Surveillance, Epidemiology, and End Results)Programm analysiert, bei denen ein
HNSCC vorlag. Um herauszufinden, ob die Ausdehnung der Lymphknotenmetastasierung ein unabhängiger prognostischer Faktor für das Überleben der Patienten ist, wurde ein multivariates CoxModell genutzt. Bei 2.463 Patienten hatte der Tumor die Mundhöhle befallen, bei 8.567 den Oropharynx, bei $2.332 \mathrm{den}$ Larynx und bei 1.137 den Hypopharynx. Sowohl die N-Klassifikation des American Joint Committee on Cancer (AJCC) als auch die Ausdehnung der Lymphknotenmetastasierung hatten einen deutlichen Effekt auf das Gesamtüberleben bei Patienten mit einem Tumor der Mundhöhle, des Oropharynx oder des Larynx, nicht aber bei Betroffenen mit einem HNSCC des Hypopharynx. Bei Patienten mit Oropharynx- oder Larynx-HNSCC im Stadium N2 war die AJCC-Subklassifikation (N2a, $\mathrm{N} 2 \mathrm{~b}$ oder N2c) signifikant mit dem Gesamtüberleben assoziiert, nicht aber bei Patienten mit einem HNSCC der Mundhöhle oder des Hypopharynx im Stadium N2. Bei Anwendung der AJCC-Klassifikationen wurden Überlappungen in den Überlebenskurven von Patienten mit verschiedenen AJCC-Subklassifikationen beobachtet, etwa N2a und N2b bei Patienten mit Mundhöhlen-HNSCC.

Fazit: Das Ausmaß des Lymphknotenbefalls ist ein unabhängiger prognostischer Faktor für Patienten mit lokal fortgeschrittenem HNSCC. Es könnte zum prognostischen Wert der T- und NKlassifikation des AJCC beitragen. Sie hat sich in dieser Studie für eine große Gruppe von HNSCC-Patienten als suboptimales Tool für die Vorhersage des Gesamtüberlebens erwiesen.

Christina Berndt

Xing $Y$ et al. Relation Between the Level of Lymph Node Metastasis and Survival in Locally Advanced Head and Neck Squamous Cell Carcinoma. Cancer. 2016;122(4):534-45. 